\title{
Attitude populiste des assureurs
}

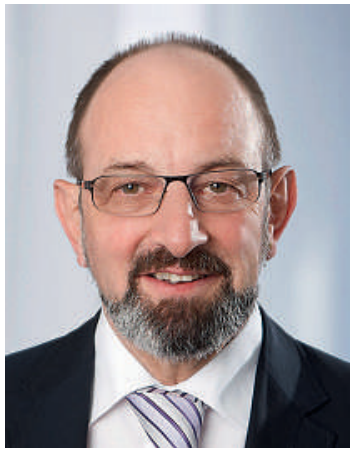

Fidèles à la devise «Ne m'assommez pas avec des chiffres, j'ai déjà ma propre idée», les assureurs tentent de faire oublier leurs propres insuffisances en se livrant à la provocation et en attaquant le projet TARVISION de la FMH dans le dernier numéro de leur organe «Mise au point». En réalité, la FMH est parvenue à rallier les derniers vrais spécialistes du TARMED et à mettre à jour les trois modèles de coûts du tarif à la prestation TARMED en tenant compte des facteurs économiques. Sans oublier les paramètres médicaux - les minutages par exemple - que nous avons actualisés dans le cadre de notre projet en collaboration avec les sociétés de discipline. santésuisse, de son côté, n'a rien fait, malgré l'urgence de la révision du TARMED.

$\mathrm{Ni}$ la FMH ni H+ n'ont parlé d'argent ou de revenus en rapport avec la révision. Car dans un premier temps, la priorité est d'actualiser la structure tarifaire, comme l'exige la loi (art. 43 al. 4 LAMal). Si l'on pense que les données économiques sur lesquelles repose le TARMED datent des années 1994-1996, même les personnes qui ne connaissent strictement rien au système de santé comprendront que l'économicité n'est plus garantie. Cette révision de la structure tarifaire - dont personne ne conteste la nécessité - est justement au cœur du projet TARVISION. Ce n'est qu'une fois qu'elle sera terminée que l'on pourra parler de prix, et plus exactement, que les sociétés cantonales de médecine pourront entamer les négociations sur la rémunération par point tarifaire.

\section{La structure tarifaire TARMED repose sur des données obsolètes - la FMH s'attelle à les mettre à jour dans le cadre de son projet TARVISION.}

La FMH dispose non seulement de ses propres chiffres, mais ces chiffres bénéficient également d'un degré de couverture particulièrement élevé. En effet, cela fait plus de sept ans que le corps médical collecte, via ses TrustCenters, des données sur la facturation et les prestations, qui sont ensuite traitées, analysées et évaluées par l'entreprise NewIndex. Ces évaluations, mises à la disposition de la FMH et de la Conférence des sociétés cantonales de médecine en vue des négociations avec les assureurs et l'OFSP, jouent un rôle essentiel dans le projet TARVISION et l'actualisation de la structure tarifaire. De plus, grâce à l'étude $\mathrm{RoKo}^{\circledR}$ (étude permanente sur les coûts des cabinets médicaux), le corps médical dispose depuis plus de 20 ans de données sur les loyers, les salaires du personnel non-médical, les intérêts, etc. Ces données montrent clairement que si le prix des loyers et des intérêts a reculé depuis l'introduction du TARMED en 2004, les salaires du personnel non-médical ont quant à eux sensiblement augmenté.

\section{santésuisse prétend s'engager pour}

\section{la revalorisation de la médecine} de premier recours, alors qu'en réalité, elle fait tout pour affaiblir la position des médecins de famille.

santésuisse affirme que les cabinets de groupe permettraient des gains d'efficacité et donc des économies importantes mais n'avance aucun chiffre pour appuyer ses dires. Or il faut savoir que les cabinets de groupe requièrent beaucoup plus de personnel que les cabinets individuels. De plus, les salaires sur lesquels reposent les modèles de coûts n'ont pas été adaptés au renchérissement depuis 1996 et devront être clairement revus à la hausse lors de la révision de la structure tarifaire. La FMH dispose de données empiriques actuelles pour étayer ses arguments.

Par ailleurs, santésuisse déclare à qui veut l'entendre qu'elle soutient la revalorisation de la médecine de premier recours. Mais jusqu'ici, on a plutôt l'impression du contraire, comme en témoigne son refus de prolonger et d'intégrer l'indemnité de dérangement en cas de visite dans le TARMED. A cause de son veto lors de l'adoption de la version 1.08 , la position 00.0065 sera suspendue à partir du 31 mai 2012 et ne figurera plus dans le TARMED. Une fois de plus, au détriment des médecins de premier recours. Mais ce n'est pas tout. Après la baisse massive des tarifs des laboratoires, santésuisse et le Surveillant des prix entendent également réduire la marge sur la vente de médicaments de $12 \%$ à 3,9\%, sans compensation. Là encore, au grand dam des médecins de premier recours.

santésuisse a peur de la vérité des coûts - mais tôt ou tard, elle sera rattrapée par la réalité économique. Toutes ces manœuvres populistes visant à dresser les médecins de famille contre les spécialistes sont donc vaines. Quoiqu'il en soit, la FMH est disposée à discuter des coûts réels et de la supposée augmentation de l'efficacité et de la productivité.

Dr Ernst Gähler, vice-président de la FMH, responsable du domaine Tarifs et Conventions 